\title{
Global Strong Solution to a Thermodynamic Compressible Diffuse Interface Model with Temperature Dependent Heat-conductivity in $1-\mathrm{D}$
}

\author{
Yazhou Chen ${ }^{1}$, Qiaolin $\mathrm{He}^{2}$, Bin Huang ${ }^{1}$, and Xiaoding Shi ${ }^{1}$ \\ ${ }^{1}$ Beijing University of Chemical Technology \\ ${ }^{2}$ Sichuan University
}

July 25, 2020

\begin{abstract}
In this paper, we investigate the wellposedness of the non-isentropic compressible Navier-Stokes/Allen-Cahn system with the heat-conductivity proportional to a positive power of the temperature. This system describes the flow of a two-phase immiscible heat-conducting viscous compressible mixture. The phases are allowed to shrink or grow due to changes of density in the fluid and incorporates their transport with the current. We established the global existence and uniqueness of strong solutions for this system in 1-D, which means no phase separation, vacuum, shock wave, mass or heat or phase concentration will be developed in finite time, although the motion of the two-phase immiscible flow has large oscillations and the interaction between the hydrodynamic and phase-field effects is complex. Our result can be regarded as a natural generalization of the Kazhikhov-Shelukhin's result ([Kazhikhov-Shelukhin. J. Appl. Math. Mech. 41 (1977)]) for the compressible single-phase flow with constant heat conductivity to the non-isentropic compressible immiscible two-phase flow with degenerate and nonlinear heat conductivity.
\end{abstract}

\section{Hosted file}

global-solution-NSFAC-1d-ib.pdf available at https://authorea.com/users/346080/articles/ 472186-global-strong-solution-to-a-thermodynamic-compressible-diffuse-interface-modelwith-temperature-dependent-heat-conductivity-in-1-d 